\title{
FOURTEEN SPECIES OF SKEW HEXAGONS
}

\author{
H. S. WHITE
}

1. Hexagon and hexahedron. For a tentative definition, let a skew hexagon be a succession of six line segments or edges, finite or infinite, the terminal point of each being also the initial point of another, the terminus of the last being the first initial point. Secondly, let there let be six marks to designate the points, the six to be in a fixed order. Further, let the first point be any one of the six, so that only the cyclic order is essential. At each of the six points, pass a plane through the two lines, and denote the plane by the same mark as that point. Adjoin to the figure these six entire planes, a complete hexahedron.

2. Sets of 64 hexagons. The initial and terminal points of an edge denote ambiguously two segments, one finite and one infinite. If the marks in their order are 123456 , then that cycle may denote equally any one of 64 different hexagons, $2^{6}=64$, obtained by selection. If desired, the one whose edges are all finite may be taken to represent the set. But we can define positive and negative directions along each line, arbitrarily on one, then by inference on all other lines of intersection of the planes. Thereafter it will be natural to understand by 12 a positive segment on the line, by 21 a negative.

3. Hexagons and cyclic permutations. As above defined, every skew hexagon determines one and only one set of six planes, but these intersect, not in 6 but in 15 lines, and so contain many different hexagons. Any cyclic permutation of the marks of the planes, as 154263 , may be interpreted as describing a hexagon. An edge is given by any four consecutive marks; for example, 2631 will denote a segment on the intersection-line 63 , from its point in plane 2 to its point in plane 1 . Thus a cycle reversed will denote the same edges in reverse order and we shall consider the two as identical. As there are 120 cyclic permutations of 123456 , this gives us only 60 different hexagons to assort into species.

All sets of 6 planes will be defined as equivalent, provided no four have a common point. By moving planes freely any one can be made to coincide with any other set, without passing through the excluded positions. It is well known that the traces of five planes in a sixth bound in that sixth one convex pentagon as well as certain triangles and quadrilaterals. Of the six pentagons, each has two edges which are edges of other pentagons, thus providing a natural order of the planes. Moreover those six common edges form a continuous 
broken line or skew hexagon, which shall be called the normal hexagon of that set of planes. Let its vertices and planes be marked, as described above, in their order along the normal hexagon, 1, 2, 3, 4, 5, 6, the vertex 1 being any one taken at random. This gives 6 equivalent notations for the set; in other words, the set of 6 planes is equivalent to itself under the cyclic permutations of the normal hexagon. Consequently, any other skew hexagon occurring in the figure is equivalent to that derived from it by cyclic permutation: $S=(123456)$, of the marks of its 6 planes and vertices. Note that the planes take their marks from the vertices of the normal hexagon, after which the vertices of any other hexagon in the figure (having two adjacent edges in each plane) take their marks each from the plane that contains the two edges at that point.

For example, 146235 will denote a skew hexagon different from the normal. The operations repeated give the 5 equivalent cycles:

$\begin{array}{llllll}2 & 5 & 1 & 3 & 4 & 6, \\ 3 & 6 & 2 & 4 & 5 & 1, \\ 4 & 1 & 3 & 5 & 6 & 2, \\ 5 & 2 & 4 & 6 & 1 & 3 \\ 6 & 3 & 5 & 1 & 2 & 4 .\end{array}$

Thus there are found sets of 6 equivalent hexagons, but also some of fewer than 6. For example 153426 is in a set of only 3.

4. Survey of all species of skew hexagons. On any single set of six planes will be found all possible species of hexagons, for any given

TABLE I

\begin{tabular}{c|c|c|c|c|c}
\hline \hline No. & In Set & Cycle & No. & In Set & Cycle \\
\cline { 2 - 5 } & 1 & 123456 & 8 & 6 & 132645 \\
2 & 6 & 146235 & 9 & 3 & 123654 \\
3 & 6 & 132654 & 10 & 3 & 135264 \\
4 & 6 & 125364 & 11 & 3 & 135462 \\
5 & 6 & 142356 & 12 & 3 & 132564 \\
6 & 6 & 143562 & 13 & 3 & 142365 \\
7 & 6 & 162345 & 14 & 2 & 143652 \\
\hline
\end{tabular}

hexagon, nonsingular, gives rise to a set of 6 planes, and all such sets are equivalent. If the planes have been referred to their normal hexagon, then all 60 hexagons that lie in all six planes are represented in Table 1 above, a single cycle representing six, or three, or two types 
of 64 that share a single set of 6 lines, or the one unique normal type.

5. A positive order on each line. Suppose the vertices of the normal hexagon to be marked in the order of their occurrence along its periphery: 123456 . Each segment, as 12, is known to be tangent at some

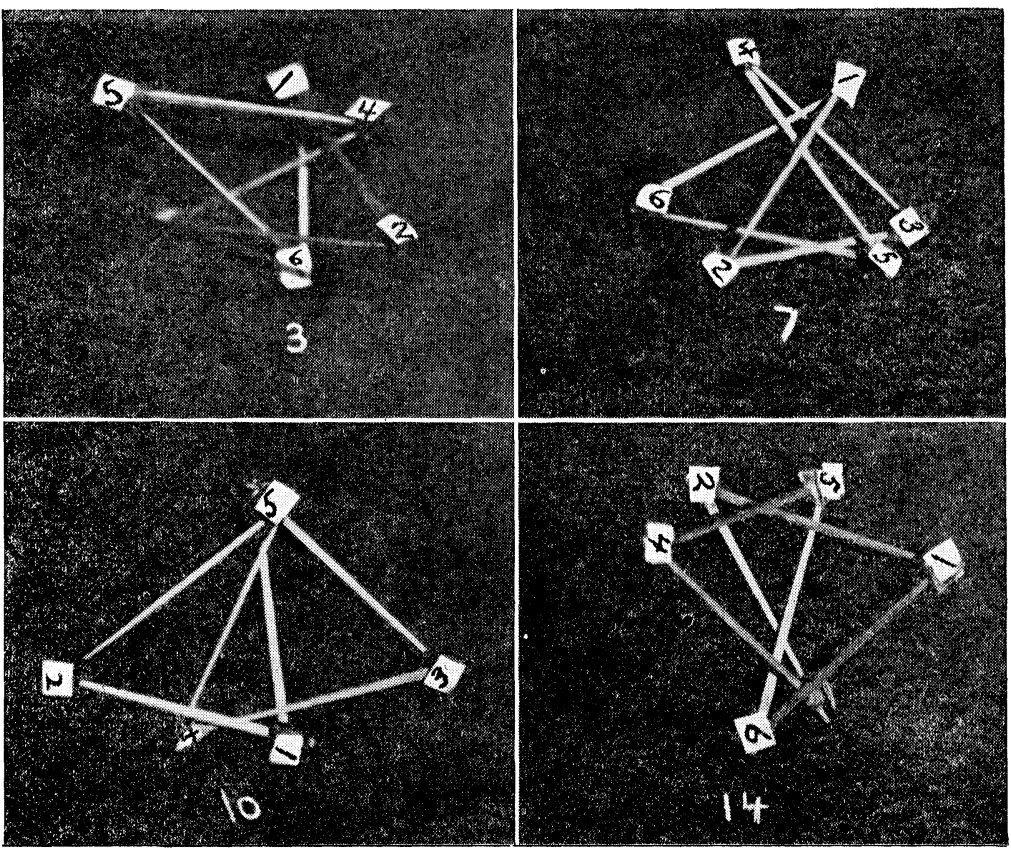

EXPERIMENTAL MODELS, CRUde BUt SERVICEABLE, OF SKEW HEXAGONS

No. 3, Normalized cycle? 143265.

No. 7, Normalized cycle? 164532.

No. 10, Normalized cycle? 146253.

No. 14, Normalized cycle? 125634.

one of its points to a conic enveloping, in plane 1, the traces of the other planes in the order, which shall be chosen as positive order, of their arrangement about the unique convex pentagon formed by those traces, say 23456, and indeed it touches on the same segment the line conics in planes 1 and 2 . Then on any other line, as 5, in plane 1 the intersections of the others must occur in the same order, 2346, omitting its own mark 5. So on all the lines in plane 1 we have a 
"normal" assignment of marks. Then since each line lies in two planes, the marking is automatically extended to all the lines in all 6 planes.

6. A character of every hexagon. On the lines of the normal hexagon the external segment must contain two intersections of other

TABLE II

\begin{tabular}{c|c|c|c}
\hline \hline Species & Character & Species & Character \\
\cline { 2 - 3 } 1 & 000000 & 8 & 120102 \\
2 & 002220 & 9 & 221001 \\
3 & 221102 & 10 & 001221 \\
4 & 001210 & 11 & 110110 \\
5 & 000112 & 12 & 211101 \\
6 & 010201 & 13 & 101112 \\
7 & 001010 & 14 & 020202 \\
\hline
\end{tabular}

planes. And since the internal segment is designated by terminals in their positive order, the external will be negative from the same initial point to the same terminus. Give it therefore two marks - and 2. Extending this scheme, assign to each directed segment a sign + or and either 0,1 , or 2 to indicate how many planes cut it internally. Every hexagon will then have six double "characters" in a definite cyclic order, the normal hexagon being described by $\left(0^{+} 0^{+} 0^{+} 0^{+} 0^{+} 0^{+}\right)$. We have now to list the characters of all 14 species for comparison.

A segment of such a hexagon being given by an ordered tetrad, as 4265 , we have yet to choose between the positive and the negative direction from 4 to 5. Always we shall take the positives for a typical representative of the 64 which share the same designation (cf. §4). With this convention, we need write only the character 0,1 , or 2 for each segment of the hexagon. In the above example, on the line 26 the marks 2 and 6 are not in intersecting planes, and the two intersections 1,3 do not fall on the positive segment from 4 to 5 . The mark will be 0 . Again, for a tetrad 2615 it is 2 , for 6413 it is 1 , and so on. For the 14 species the characters of the representative hexads are shown in Table II.

These characters are not quite distinctive, but nearly so. Sets 3 and 5 differ as right-hand and left-; also sets 12 and 13. But not so sets $8,9,10$, and 14: each is auto-symmetric, and a change of sense will not exchange them. Distinguish odd and even triads of planes, and we see that triads are collected in No. 10, alternated in sequence in No. 9.

There are, therefore, 14 types among these 60 skew hexagons, all lying 
in the same set of 6 planes, two segments in each plane. No two types are similar as regards distribution of intersection-points; but two pairs are symmetrical to each other, and four sets are auto-symmetric.

THEOREM. The character of every skew hexagon must contain no aces, or two, or four. (A synthetic proof seems desirable.)

7. Determining the species of a concrete model. When a given material model is presented for classification, one first assigns marks, as 123456 to the vertices in their order along the perimeter, first point and direction being chosen at random. Next, the cyclic order of points on each side is found by direct observation. From this, the determination of a normal order is most readily shown by an example.

From a certain specimen we take these sequences:

$\begin{array}{cccc}\text { On line } & \text { Order } & \text { On line } & \text { Order } \\ 12 & 6453 \text { or } 6354 & 45 & 3162 \text { or } 3261 \\ 23 & 1654 \text { or } 1456 & 56 & 4321 \text { or } 1234 \\ 34 & 2615 \text { or } 2516 & 61 & 5423 \text { or } 5324\end{array}$

To unite these consistently in a hexad, take the first and third. They require 264153 or 261453 . Comparison with the order on 23 decides in favor of 261453. Adopt this for normal order. Apply the substitution:

$$
T=\left(\begin{array}{l}
261453 \\
123456
\end{array}\right) \text {, }
$$

by which the normal order becomes 123456 . This redesignates the points, and the given hexagon is now entitled $T(123456)=316452$. This has the character 202121. As this is not found in Table II, adopt the reverse normal order, and find for character 020101. This is No. 6 on the list. Verify by applying the operator $S=(123456)$ five times to 316452 . The last member of the set is 265341 , readily recognizable as the reverse of No. 6 which was 143562 . As errors are easy in calculating the character, such a final check should always be demanded. Another example, if desired for practice, is taken from a second model. The orders observed are: $6543,5146,2156,3612,4132,5324$, leading to No. 8 in the table.

This plan of classification is hardly practicable for skew $n$-gons when $n>6$, owing to the rapid increase in number of species. For a single species of $n$-gon, whose vertices are on a twisted cubic curve, it could be generalized.

Vassar College 